\title{
100-GHz Grid-Aligned Multi-channel Polarization Insensitive Black-Box Wavelength Converter
}

\author{
Víctor J. F. Rancaño, Francesca Parmigiani, Periklis Petropoulos, Senior Member, OSA \\ and David J. Richardson, Fellow, OSA
}

\begin{abstract}
A new scheme for a flexible modulation and bit rate independent, polarization-insensitive optical wavelength converter (WC) based on four-wave mixing (FWM) in a highly nonlinear fiber (HNLF) is demonstrated and characterized for different modulation formats and data rates. Its robustness is demonstrated in a data transmission experiment in which the WC is incorporated at the mid-point of a transmission line based on installed fiber. In addition, an optical feedback system designed to prevent slow polarization drift of the pumps and consequently to ensure the long-term stability of the system is implemented and characterized. The WC is tested with the feedback system in place enabling turn-key operation.
\end{abstract}

Index Terms - nonlinear optics, four wave mixing, wavelength conversion devices

\section{INTRODUCTION}

$S_{a}$ IGNIFICANT advances have been achieved in the field of all-optical wavelength conversion over the last decade, driven mainly by its importance in avoiding optical-to-electronic-to-optical (OEO) conversion in reconfigurable optical add-drop multiplexers (ROADMs) and the need to ensure more efficient use of the spectrum in fiber networks [1,2]. However, despite its importance, most work reported in the literature to date has focused on optimizing certain features of WCs [3-8] at the expense of others, rather than taking a holistic approach and ensuring that all features required of a practical network device are considered and incorporated.

For example, some schemes allow for a broad operational bandwidth, free of high-order mixing components, but only at the expense of low gains $[3,4]$; others maximize the conversion gain, neglecting the need for polarization insensitivity $[5,6]$ and different approaches allow very low conversion power penalties but are not compatible with the standard ITU frequency grid $[7,8]$. Nevertheless, for a WC to be deployed in real systems, it

Manuscript received 5 Nov, 2013. This work was supported by the EPSRC grant EP/I01196X: Transforming the Future Internet: The Photonics Hyperhighway. Dr F. Parmigiani is supported by a Royal Academy of Engineering/EPSRC research fellowship.

V. J. F. Rancaño, F. Parmigiani, P. Petropoulos and D. J. Richardson are with the Optoelectronics Research Centre, University of Southampton, Southampton, SO17 1BJ, UK. (e-mail: vjfr1u10@orc.soton.ac.uk, frp@orc.soton.ac.uk,pp@orc.soton.ac.uk,djr@orc.soton.ac.uk). is important that it satisfies all the requirements mentioned above simultaneously. Furthermore, all-optical wavelength conversion schemes should be able to support multichannel operation in order to be economically viable and to improve their practicality.

Developing a scheme capable of black-box operation in a real communication network involves establishing a trade-off between all the aforementioned features: broad bandwidth, high gain, insensitivity to both polarization and modulation format, ease of filtering and multichannel operation.

In this work, we present a novel, flexible WC scheme, which seeks to provide a compromise amongst all these requirements and characterize its performance for a $100-\mathrm{GHz}$ grid-aligned implementation. Its configuration provides sufficient flexibility to allow it to be directly incorporated in an optical add-drop multiplexer (OADM), or in a ROADM. Its operation is based on FWM in a HNLF, pumped by two orthogonally polarized, widely frequency-spaced beams. The $100-\mathrm{GHz}$ grid-aligned WC showed a flat conversion gain (defined in terms of the signal power at the black-box WC output versus the input signal power) over $10 \mathrm{~nm}$ that ranged between 0 and $9 \mathrm{~dB}$ (depending on the input signal power), a polarization dependent loss (PDL) of $<0.64 \mathrm{~dB}$ and a conversion penalty of $<2.5 \mathrm{~dB}$ for on-off keyed (OOK), binary phase shift keyed (BPSK), quadrature phase shift keyed (QPSK) and 16-quadrature amplitude modulation (16-QAM) signals working at data rates of up to 40 Gbit/s [9].

We characterized the performance of the WC in a $1200-\mathrm{km}$ transmission experiment over installed fiber. The WC, configured as a black-box device, was placed at the mid-point of the fiber link (part of the UK's Aurora dark fiber network [10]) and its performance in transmission was measured to be essentially identical to that achieved in our earlier lab-based experiments, even in the case of multichannel operation [11]. Finally, a feedback system was introduced, which corrected for slow drifts of the relative states of polarization of the two pumps and ensured that they remained orthogonally polarized over extended (effectively unlimited) periods of time.

The paper is organized as follows: in Section II, we describe the WC scheme and characterize it under single-channel operation for various modulation format signals. In Section III, we assess its performance in both single- and multi-channel operation with the device placed within an installed fiber link. In Section IV, the implementation of the automatic polarization 
alignment feedback system is described and characterization data confirming correct and reliable operation is presented. Finally, we draw our conclusions in Section V.

\section{WAVELENGTH CONVERTER SCHEME}

Fig 1 shows the schematic of the WC. It incorporates two arrayed waveguide gratings (AWGs) to combine the incoming data signals to be converted with two orthogonally polarized pump beams (with linewidths of about $100 \mathrm{kHz}$ each) at the input of the HNLF, and an output filter to allow simultaneous re-transmission of only the generated idlers. The scheme presents the following key characteristics: (i) polarizationinsensitive operation with respect to the input signals, ensured by the two orthogonally aligned pumps; (ii) easy filtering of the converted signals, allowed by the wavelength allocation of the two pumps, which ensures that any polarization-sensitive mixing components are separated with high extinction from the polarization-insensitive idlers; (iii) high and flat conversion gain across the whole conversion band, guaranteed by the use of a dual-pump FWM process in conjunction with a stateof-the-art HNLF; (iv) grid-aligned, black-box operation, allowed by the combination of an erbium-doped fiber amplifier (EDFA) at the WC input, followed by an AWG; finally, (v) multi-channel operation as the system is resilient to high-order (HO) mixing components. Furthermore, the undesired HO mixing components that are not at the converted signal wavelengths can be easily filtered out at the output of the system, so that new signals can be added within the WC output bandwidth (at different wavelengths as compared to the converted ones) without extra penalty. Indeed, the WC output can be fed to a wavelength-selective switch (WSS) for filtering and combined with other channels, as will be described in the following section, which is the standard procedure used in ROADM architectures.

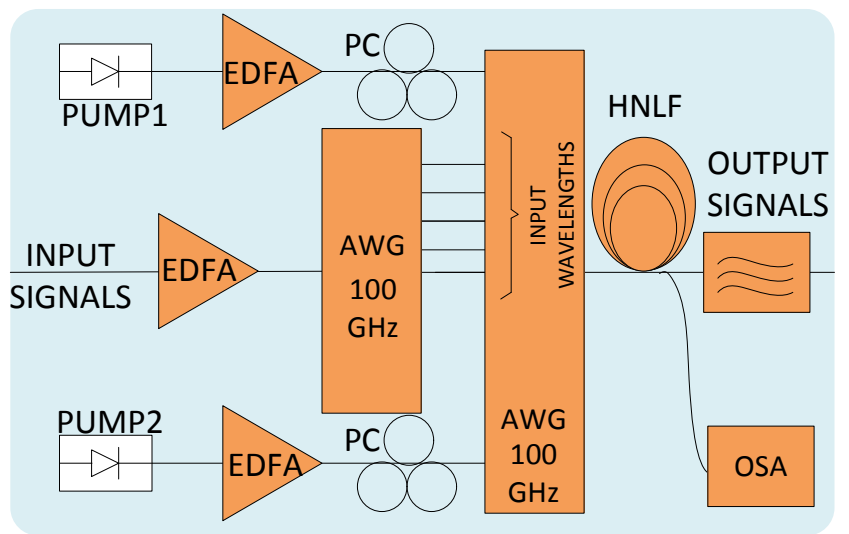

Fig. 1. Wavelength converter set-up. EDFA: Erbium-doped fiber amplifier, AWG: Arrayed waveguide grating, HNLF: Highly nonlinear fiber, PC: polarization controller, OSA: optical spectrum analyzer.

At the WC input, the various signals were amplified up to a total power of $12 \mathrm{dBm}$ and filtered by the AWGs in order to remove amplified spontaneous emission (ASE). The initial removal of ASE within the bandwidth of the converted signals is critical, since without this the conversion power penalty is increased by $5-6 \mathrm{~dB}$. The channelized filtering characteristics of the AWG allow a single amplifier to be used even for operation with multiple channels. The second AWG also facilitates filtering of the ASE of the pumps, which were amplified so that the power of each of them was $21.5 \mathrm{dBm}$ at the HNLF input. It is to be noted that while the use of the first AWG is not needed for single channel operation, it is essential for multi-channel operation. Therefore, it has been included in the various measurements presented in this paper, to emphasize the flexibility of the scheme.

At first, the polarization alignment of the two pumps was performed manually by adjusting their corresponding polarization controllers (PCs) depicted in Fig. 1, monitoring and minimizing their mutual nonlinear interaction using an optical spectrum analyzer (OSA). Subsequently, we automated this polarization alignment, as we will discuss in Section IV. The $302 \mathrm{~m}$-long HNLF used was a germanium-silicate dispersionshifted strained fiber with a nonlinear coefficient of 11.6 $(\mathrm{W} \cdot \mathrm{km})^{-1}$, a dispersion slope of $0.018 \mathrm{ps} /\left(\mathrm{nm}^{2} \cdot \mathrm{km}\right)$ and an average zero-dispersion wavelength (after straining) of 1555 $\mathrm{nm}$. The strain gradient was applied along the HNLF to broaden the Brillouin gain bandwidth and reduce its peak gain, thereby increasing its stimulated Brillouin scattering (SBS) threshold to $27 \mathrm{dBm}$ [12]. Finally, the (flat-top) coarse output filter was tuned to cover the entire output bandwidth.

Significant performance degradation due to FWM-induced pump depletion in the HNLF was avoided by keeping the EDFA used for the amplification of the input signals in automatic power control (APC) mode. In this mode, the overall output power of the EDFA remained constant, so that a pump-to-signal(s) ratio of at least $15 \mathrm{~dB}$ was maintained at the input of the HNLF.

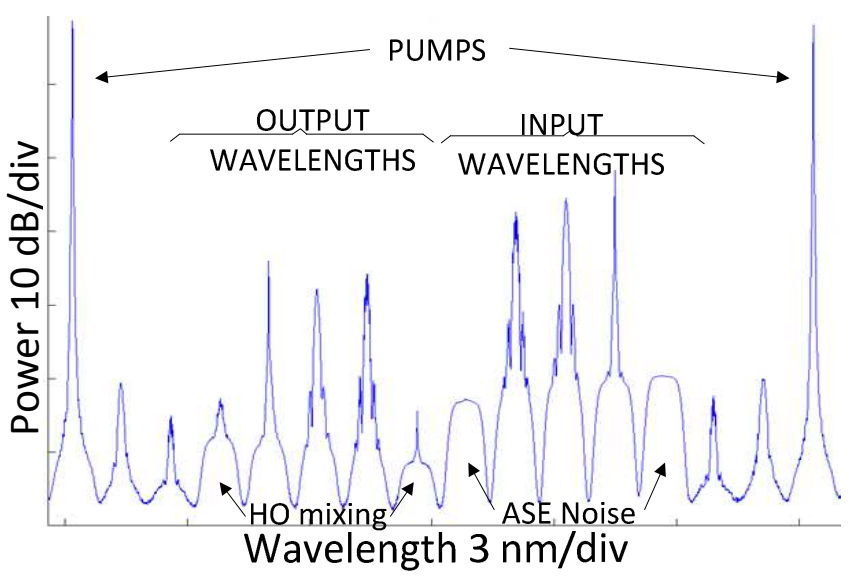

Fig. 2. An example of a multi-channel nonlinear interaction, as observed at the output of the HNLF. HO mixing: Higher order mixing. ASE noise: Amplified spontaneous emission noise.

An example of a typical nonlinear interaction for a mixed signal scenario (one OOK, one BPSK and one QPSK signal) is shown in Fig. 2, where the optical spectrum at the output of the HNLF is plotted. Due to the equal spacing between the input signals some of the higher order (HO) mixing components were generated at the same frequencies as the output signals. Nevertheless, if all the input channels have similar input 
powers, the level of cross-talk from these components can be kept below $20 \mathrm{~dB}$, causing negligible signal degradation in multi-channel operation.

In our implementation, the WC bandwidth was ultimately determined by the bandwidth of the AWGs. This band of wavelengths where the converted signals could be allocated, was about half the spacing between the two pumps and could easily be tuned by reconfiguring the connections of the AWG input ports for the pump beams. For this characterization, the two pumps were set at $1562.23 \mathrm{~nm}(191.90 \mathrm{THz})$ and 1550.12 $\mathrm{nm}(193.30 \mathrm{THz})$, and the input channels were tuned between $1559.79 \mathrm{~nm}(192.2 \mathrm{THz})$ and $1556.55 \mathrm{~nm}(192.60 \mathrm{THz}) . A$ 1 -nm guard band was used around the pump wavelengths to facilitate filtering of the idlers.
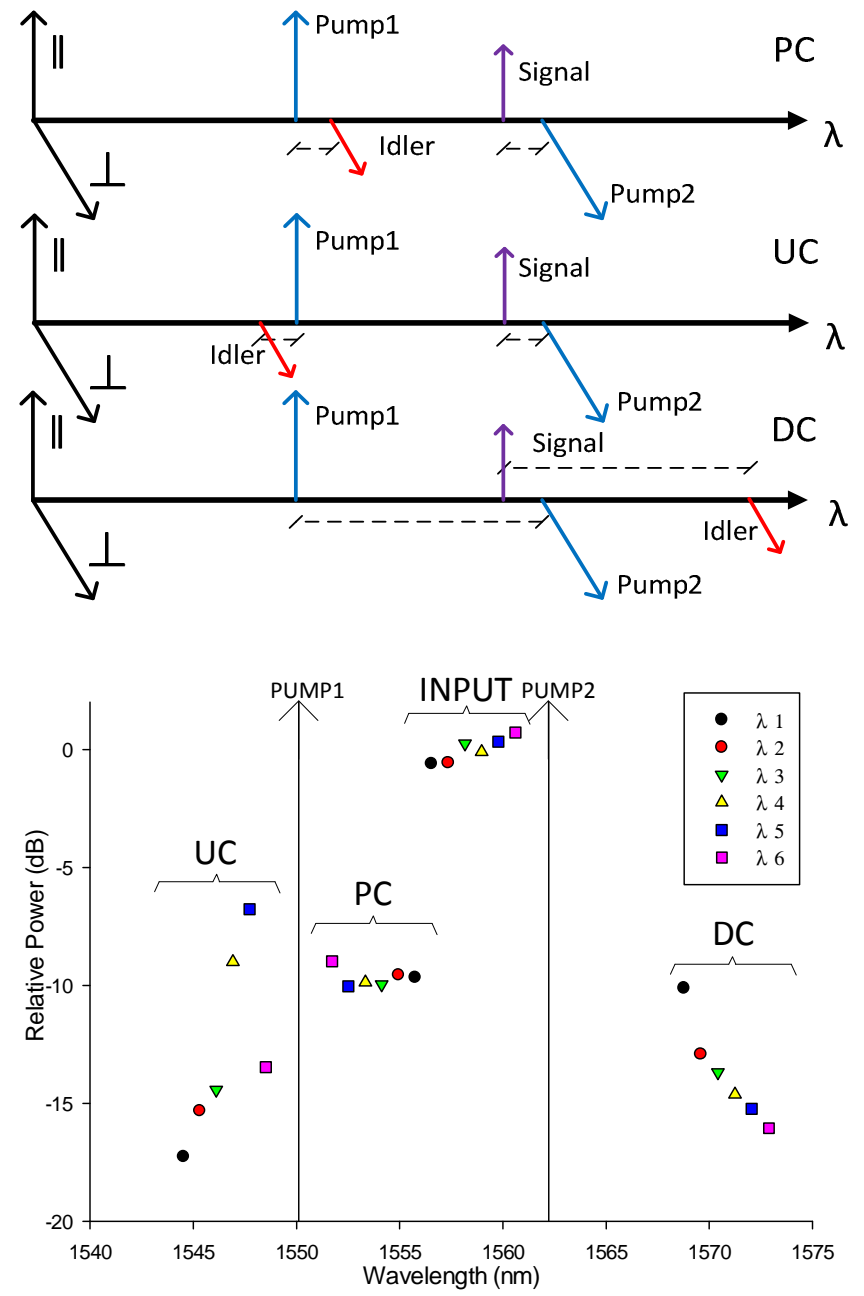

Fig. 3 Top: Schematic representation of the phase-conjugated (PC), up-converted (UC) and down-converted (DC) first-order FWM processes in the HNLF. (Note that the signal polarization needs not be aligned to that of the pumps). Bottom: Characterization of the conversion efficiencies at the output of the HNLF for the three different types of idlers as a function of the wavelength of the signals.

In general, a dual-pump parametric interaction can generate three distinct first-order FWM processes referred to as: phase conjugation (PC), Up-Conversion (UC) and Down-Conversion (DC)[13]. These processes are sketched in Fig. 3 (Top) for the case of orthogonally polarized pumps. For the sake of clarity, the signal is drawn co-polarized with pump 1 in this sketch, however it is to be appreciated that its polarization can be arbitrarily set. Figure 3 (Bottom) reports the characterization of these FWM interactions in the HNLF in terms of relative power of the wavelength converted signals as a function of the signal wavelength. The normalized signal power at the input of the HNLF is also shown for completeness. The figure shows a flat conversion gain (1 dB gain fluctuation) for the PC process. In contrast, the gain of the UC and DC processes depend strongly on the signal wavelength and exhibit a variation of more than 6 $\mathrm{dB}$ within the conversion band we studied, due to the fact that phase matching plays a critical role in these interactions. On the other hand, the frequencies of the PC idlers are mirrored (and their phases conjugated) with respect to the input, whereas the UC and DC processes do not conjugate the input. Phase conjugation of the idlers does not constrain reception of any single-channel modulation format, although it does prevent transparent wavelength conversion of multicarrier modulation formats (since the subcarrier allocation is inverted during the parametric interaction).

In our work, the PC idlers were chosen as output signals due to their constant conversion gain across the signal bandwidth rendering them suitable for multi-channel operation. Therefore, the converted signal wavelengths lay between $1555.75 \mathrm{~nm}$ $(192.70 \mathrm{THz})$ and $1552.52 \mathrm{~nm}(193.10 \mathrm{THz})$.

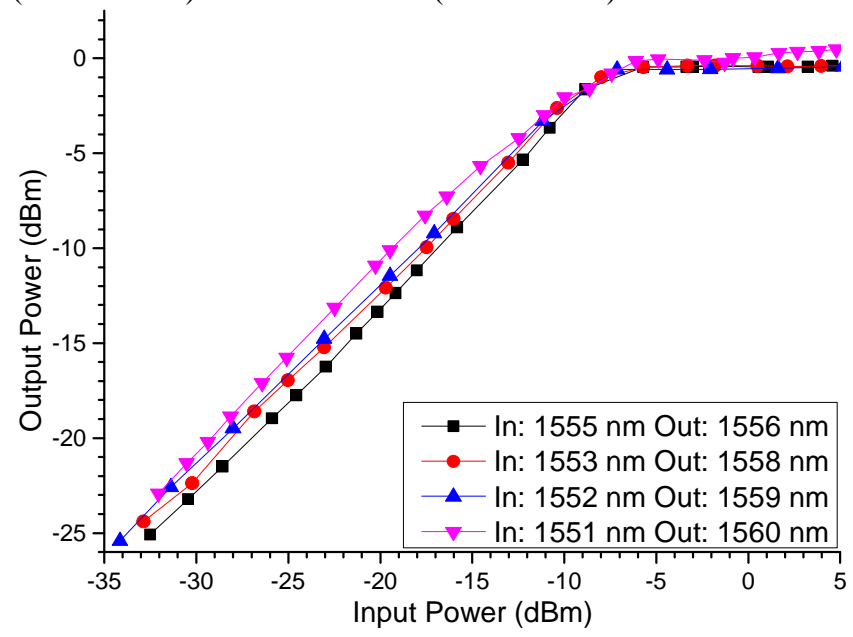

Fig. 4 Wavelength converted signal output power as a function of the signal input power at the EDFA input for various input signal wavelengths. Only one signal is considered at a time.

We next characterized the wavelength converted output power of the whole black-box WC system (which includes the EDFA, two AWGs, the HNLF and the output filter) for the PC idlers as a function of the input signal power. This measurement was repeated for several input signal wavelengths (Fig.4). Since the EDFA operated in APC mode and as long as we operate in the small signal regime, the signal power at the input of the HNLF changed in step with the power at the input of the WC system. Then two different regimes could be identified in the operation of the WC, which in turn were mainly dependent on the EDFA operation: (i) a linear output power regime (constant gain regime), corresponding to input signal powers for which 
the EDFA operated in a linear fashion and (ii) a constant output power regime, corresponding to input signal powers for which the EDFA operated in gain saturation. In the first operating regime, the power of the wavelength converted signals varied as a function of the input signal wavelength by $2 \mathrm{~dB}$, mainly due to the non-uniform gain of the EDFA across these wavelengths. The small power variations (less than $0.5 \mathrm{~dB}$ ) observed in the second operating regime, were due to non-uniformities in the insertion loss of the various ports of the AWGs, which were later shown to have a negligible effect on the system performance. It is worth emphasizing again that the pump-to-signal ratio was at least $15 \mathrm{~dB}$ for all the various input signal powers, so that significant performance degradation due to FWM-induced pump depletion in the HNLF was avoided. In all the subsequent experiments the input power of the various signals was configured so that the WC operated in the constant output power regime and yielded a conversion gain of $+1 \mathrm{~dB}$.

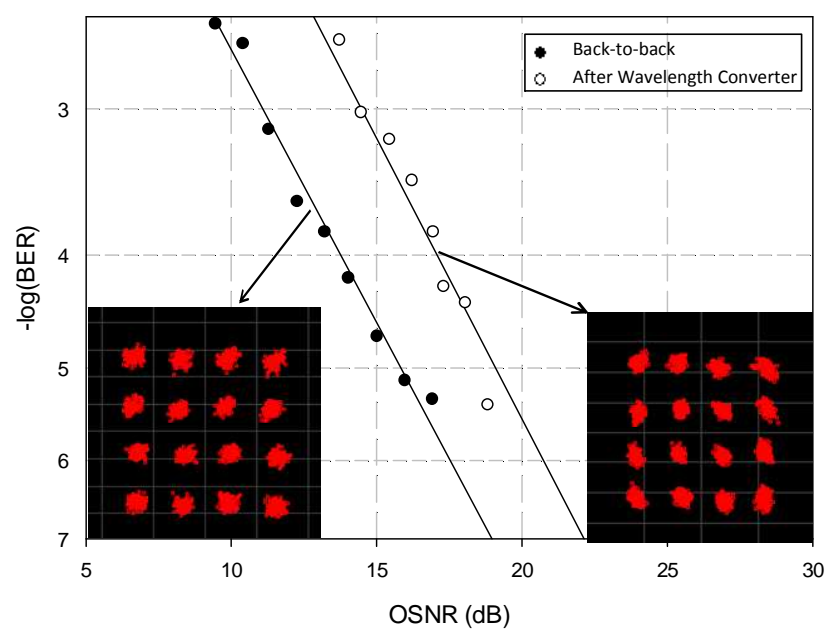

Fig. 5 BER curves and constellation diagrams for the original (full circles) and wavelength converted (empty circles) 10 Gbd 16-QAM signal.

The polarization sensitivity was characterized with a slowly varying polarization scrambler (polarization modulation frequency of about $1 \mathrm{kHz}$ ) connected at the input of the $\mathrm{WC}$ and the response to a continuous wave $(\mathrm{CW})$ signal was monitored on a digital scope. Measurements were carried out over hundreds of seconds and showed a peak-to-peak PDL of $0.64 \mathrm{~dB}$

Finally, we measured the conversion penalty of the WC for different signal modulation formats (OOK, BPSK, QPSK and 16-QAM), repetition rates (10 to $40 \mathrm{Gbit} / \mathrm{s})$ and various wavelengths. The WC showed similar performance in all of the cases we examined, and a power penalty at the receiver below $2.5 \mathrm{~dB}$ (at a bit error ratio (BER) of $10^{-5}$ ). An example of the BER curves as a function of the optical signal-to-noise-ratio (OSNR) obtained for the original (back-to-back) and wavelength converted $10 \mathrm{Gbd}$ 16-QAM signal at $1557.36 \mathrm{~nm}$ is displayed in Fig. 5, together with the corresponding constellation diagrams.

\section{FIELD TRIAL EXPERIMENTS}

After having characterized the WC as a stand-alone device, the system was introduced in the middle of an installed fiber link (the UK's Aurora dark fiber network) to assess its performance as a black box device (see Fig. 6). Six data channels were used in the transmission experiments, three of which were wavelength converted after transmission over $600 \mathrm{~km}$, coupled with the original six channels and re-transmitted over the same distance. The fiber link comprised twelve spans, as shown in Fig. 6, with distances ranging between 40 and $60 \mathrm{~km}$ each. The chromatic dispersion in the link was not fully compensated in order to optimize the OSNR achieved at the receiver. Instead, a receiver with chromatic dispersion estimation and compensation was used to properly receive and characterize the signals after the first loop and after the WC. Note, however, that digital dispersion compensation was not required for the WC signals after the second passage through the loop, since the PC idlers conjugated the phase of the original signal at the mid-point of the system after the first circulation, therefore resulting in close to zero net dispersion after the second circulation in the same fiber link [14].

In order to test the performance and robustness of the setup, two distinct network scenarios were considered, involving the simultaneous conversion of three channels, which were

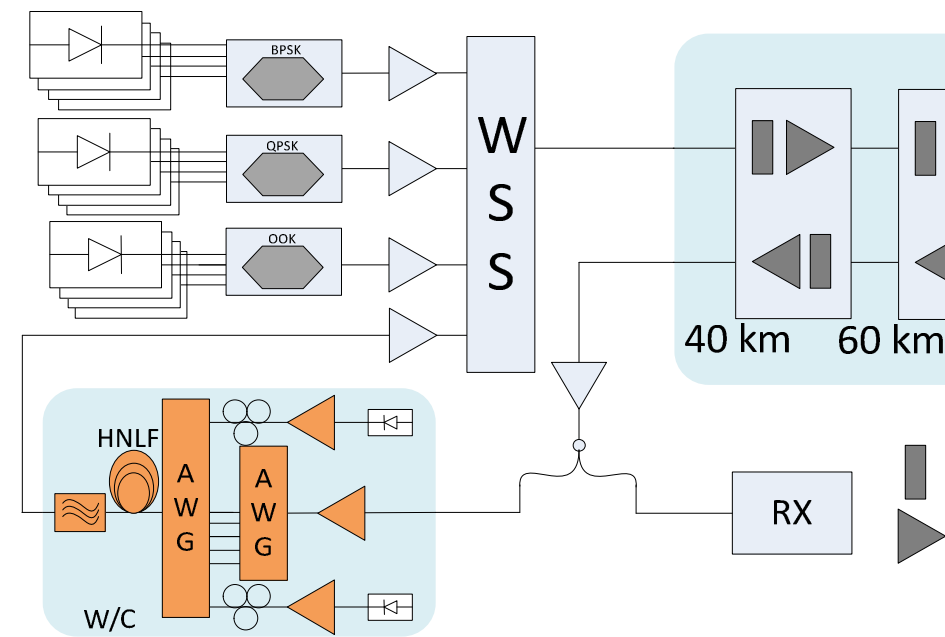

Chromatic Dispersion Compensation Module

Erbium Doped Fiber Amplifier

Fig. 6 Experimental set-up of the transmission experiment. Rx: receiver. WSS: wavelength selective switch 
dynamically reconfigured thanks to the flexibility provided by the WSS. Different modulation format signals at different wavelengths and bit rates were considered in each case, aiming at highlighting different features of the WC.

Three different modulators were used at the transmission side to generate $10 \mathrm{Gbd}$ OOK, BPSK and QPSK signals, modulated using a $2^{31}-1$ pseudo-random binary sequence (PRBS). (It is noted that the high optical signal-to-noise ratio requirements of 16-QAM modulation prevented us from using the signals of Fig.5 in any of the transmission experiments). The use of a partially dispersion-compensated transmission line ensured that the signals at the various wavelengths appeared decorrelated when reaching the WC. The signals were amplified and filtered using a WSS with a filter bandwidth of $50 \mathrm{GHz}$. The launched power per channel at the fiber link input was $2 \mathrm{dBm}$. Following wavelength conversion and after traversing the loop a second time, the converted signals were received, characterized in terms of constellation diagrams and BER and their performance was compared to that of the signals both after a first circulation around the loop and after passage through the WC.

\section{Multi-Channel Operation: Scenario 1}

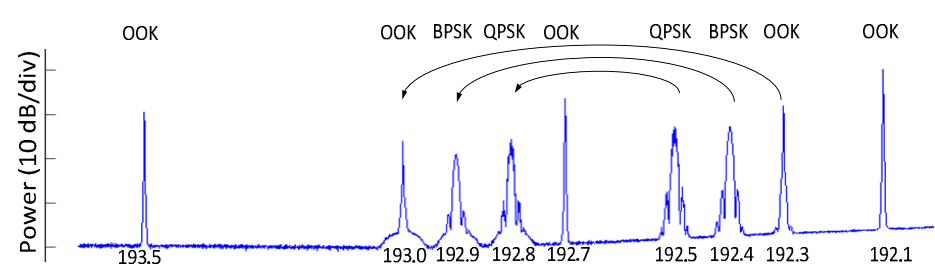

Fig. 7 Spectral allocation of the various signals at the output of the transmission link for Scenario 1. Frequency references are given in $\mathrm{THz}$.

The first scenario we considered highlights the modulation-independence and the multichannel operation of the scheme. In this scenario four non-return-to-zero (NRZ)-OOK signals at frequencies 192.1, 192.3, 192.7 and $193.5 \mathrm{THz}$, one NRZ-BPSK signal at $192.4 \mathrm{THz}$ and one NRZ-QPSK signal at 192.5 THz were transmitted through the link. The rate of all signals was $10 \mathrm{Gbd}$. The signals at $192.3,192.4$ and $192.5 \mathrm{THz}$ were chosen to be converted to $193.0,192.9$ and $192.8 \mathrm{THz}$, respectively, as shown in Fig. 7. One of the non-converted OOK signals was intentionally located in an unused part of the WC output bandwidth to prove negligible channel interference after further transmission.

The OSNRs required to achieve a BER of $10^{-3}$ for the signals that were wavelength converted are summarized in Fig. 8 for the back-to-back (B2B), after the first transmission $(600 \mathrm{~km})$, the wavelength conversion $(600 \mathrm{~km}+\mathrm{WC})$ and the second loop transmission $(600 \mathrm{~km}+\mathrm{WC}+600 \mathrm{~km})$. This figure shows that the OSNR degradation due to wavelength conversion (the difference between circles and up-triangles in Fig. 8) was very similar for the cases of BPSK and QPSK signals and was of the order of $2 \mathrm{~dB}$.

It is to be noted that the coherent receiver used at the time of the measurements did not support OOK signals as a possible modulation format, so BER measurements could only be carried out at the end of the second loop, where digital dispersion compensation was not required. For this case only, direct detection was used. Examples of eye diagrams and constellation diagrams observed at the input and at various transmission points are plotted in Fig. 9 for OOK signals (top row), BPSK (middle row) and QPSK signals (bottom row).

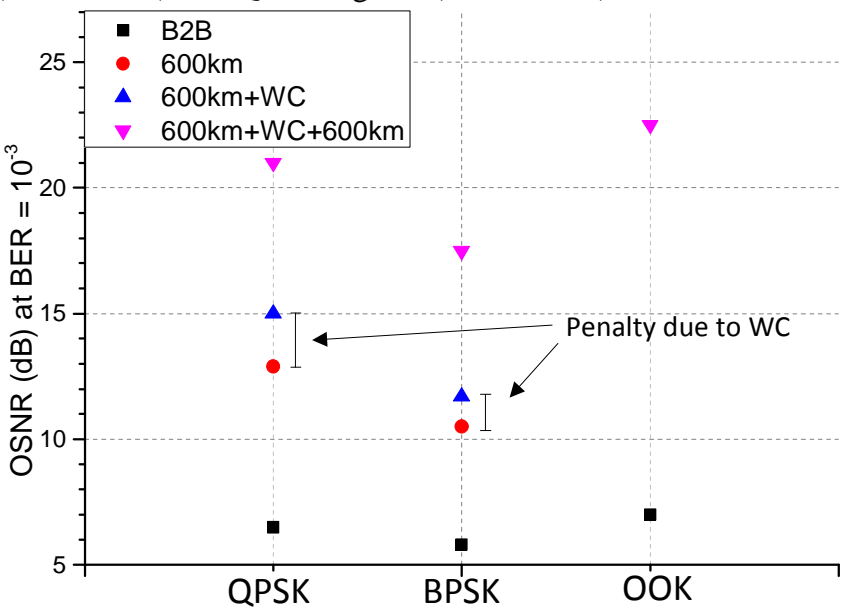

Fig. 8 OSNR values for a BER of $10^{-3}$ at different transmission points for scenario 1.

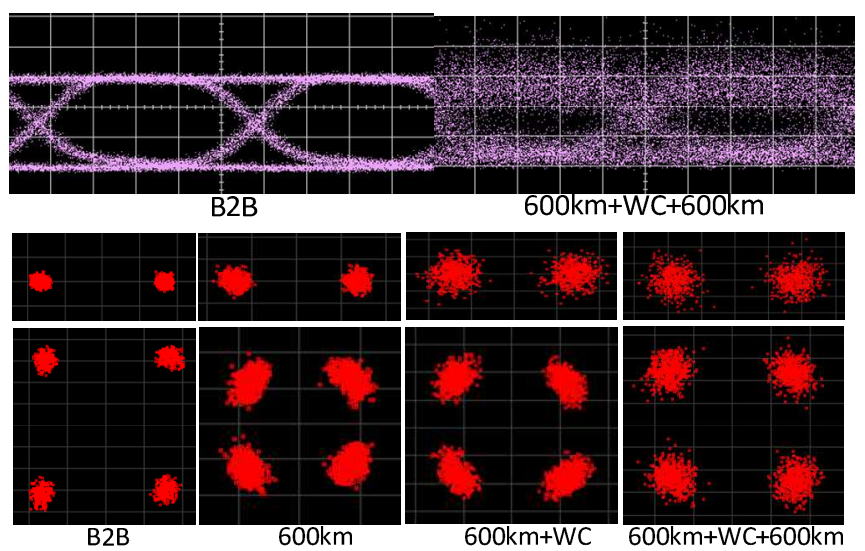

Fig. 9 Eye diagrams for the OOK signal (top row - time scale: $20 \mathrm{ps} / \mathrm{div}$ ) and constellation diagrams for BPSK (middle row) and QPSK (bottom row) signals at different transmission points for scenario 1 .

\section{Multi-Channel Operation: Scenario 2}

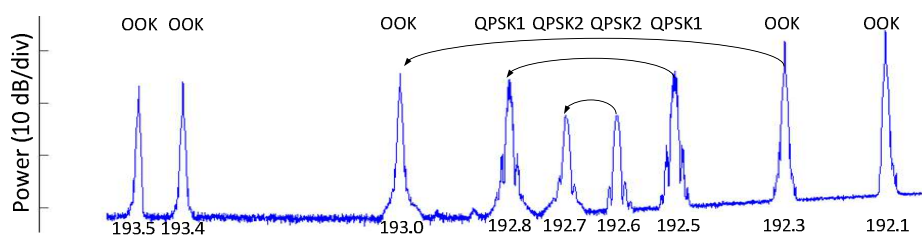

Fig. 10 Spectral allocation of the various signals at the output of the transmission link for Scenario 2. Frequency references are given in THz.

The second scenario highlights both the wavelength selectivity and multichannel conversion of the scheme. In this scenario the same four NRZ-OOK signals as in the previous case, as well as two NRZ-QPSK signals at frequencies 192.5 THz (QPSK1) and 192.6 THz (QPSK1) were transmitted through the link. The symbol rate of all signals was again $10 \mathrm{Gbd}$. The signals at $192.3,192.5$ and $192.6 \mathrm{THz}$ were chosen 
to be converted to $193.0,192.8$ and $192.7 \mathrm{THz}$, respectively as shown in Fig. 10.

As before, the OSNRs required to achieve a BER of $10^{-3}$ for the signals that were wavelength converted are summarized in Fig. 11 for the back-to-back (B2B), after the first transmission $(600 \mathrm{~km})$, the wavelength conversion $(600 \mathrm{~km}+\mathrm{WC})$ and the second loop transmission $(600 \mathrm{~km}+\mathrm{WC}+600 \mathrm{~km})$. As discussed previously, the OOK signals were not characterized at the intermediate stage, however their performance after the second passage through the loop was similar to that in Scenario 1.

It is to be noted that, in this scenario, the OSNR degradation undergone by QPSK2 was over $2.5 \mathrm{~dB}$. This was a consequence of the lower power used for this signal as compared to the remaining signals at the input of the WC. This can also be appreciated in Fig. 10, showing a power difference of $\sim 5 \mathrm{~dB}$ between the two signals, QPSK1 and QPSK2. As mentioned earlier, under these conditions the corresponding power ratio between QPSK2 and the generated HO mixing was lower than $20 \mathrm{~dB}$, causing an increase in the power penalty for that particular channel. The corresponding constellation diagrams for QPSK2 are shown in Fig.12.

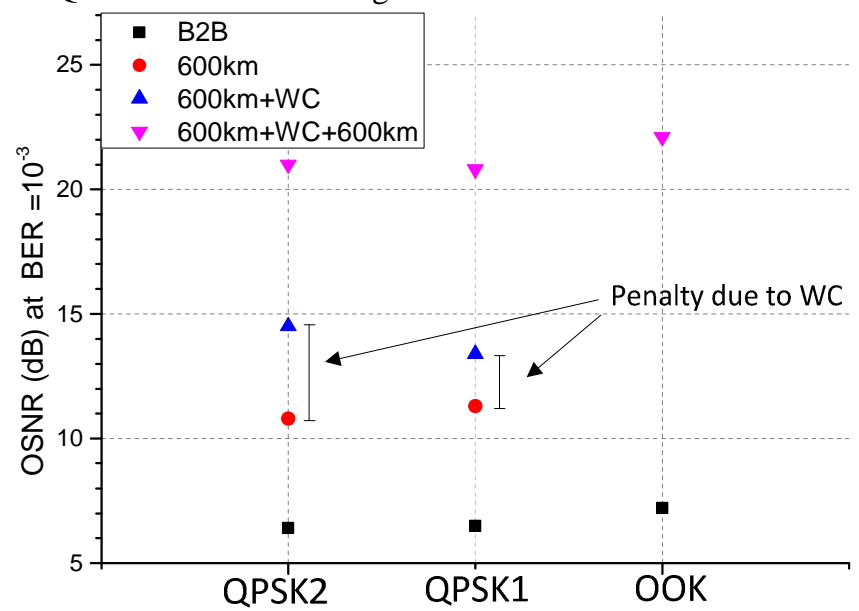

Fig. 11 OSNR values for a BER of $10^{-3}$ at different transmission points for scenario 2 .

\section{AUTOMATIC PUMP POLARIZATION ALIGNMENT}

As mentioned earlier, during the aforementioned tests the polarization adjustment of the two pumps was performed and maintained manually using the polarization controllers depicted in Fig. 1. This alignment was susceptible to vary with time due to the slow and random polarization drifts induced by temperature changes and mechanical vibrations [15-17], making it an impractical solution for real communication networks. This motivated the analysis and development of an automatic pump-alignment stabilization mechanism that is reported in this Section.

In order to overcome the polarization sensitivity of FWM-based schemes, several approaches have been reported in the literature. For example, the large polarization dependent gain (PDG) of single-pump FWM schemes in low-birefringence HNLFs can be compensated for through the use of polarization tracking devices to dynamically align the polarization of the input signals to that of the pump. However, in multi-channel operation, even if a polarization tracker was to be used for each channel (with significant implications for the overall system cost) the dependence of birefringence with respect to the wavelength might lead to different conversion gains for each input signal, thereby reducing its potential functionality.

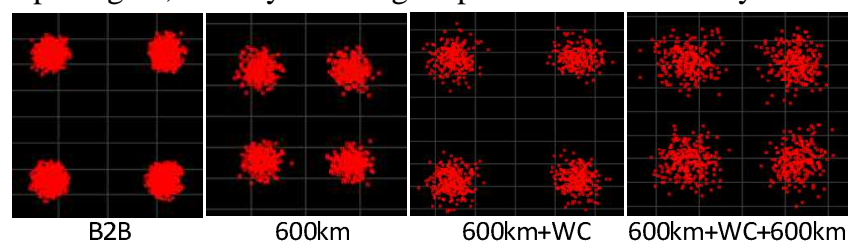

Fig. 12 Constellation diagrams for QPSK2 at different transmission points in for scenario 2 .

On the other hand, if a high-birefringence HNLF was to be used in a single-pump FWM approach to allow polarization insensitive operation of the parametric process, the differential group delay (DGD) effects induced on the converted signal(s) would restrict its practical implementation in high-speed systems [18].
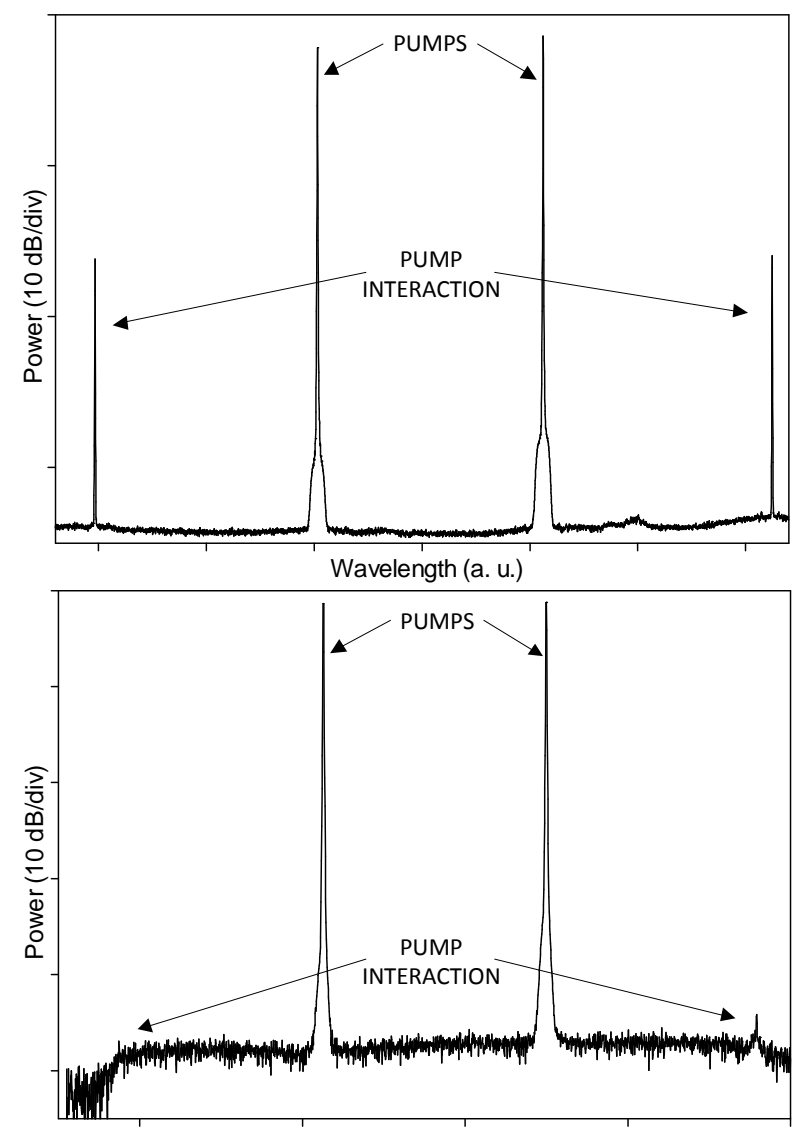

Wavelength (a. u.)

Fig. 13 Degenerate nonlinear pump interaction for a random (top) and optimum (bottom) alignment of the pumps.

In a dual-pump FWM scheme, such as the one we have considered in this work, where orthogonally-polarized pumps are employed to ensure operation that is insensitive to the signal 
polarization, the difficulty lies in maintaining the pump orthogonality at the input over time and along the length of the HNLF $[3,15]$. Thus, the outcome of the wavelength conversion is determined by averaging all the possible relative alignments of the pumps during the propagation in the HNLF, assuming that the polarization of each pump can follow a three-dimensional random walk over the Poincaré sphere [16]. Note that this evolution can vary in time due to drifts in environmental factors, such as temperature, strain and acoustic perturbations.

In order to overcome this limitation, we modified our implementation of Fig. 1 to include a feedback circuit that monitored just the performance of the nonlinear interaction between the two pumps in the HNLF without interfering with either of the original or the converted signals. The feedback circuit then dynamically readjusted the polarization of one of the pumps to maintain the desired performance over time.

Since a low-birefringence HNLF was considered (with a PMD of $0.58 \mathrm{ps} \cdot \mathrm{km}^{-0.5}$ ), each pump did not have to be aligned to a preferred fiber polarization axis and only the relative polarization alignment between the two pumps had to be controlled and adjusted. The average power of the degenerate idlers generated by the nonlinear interaction in the HNLF between the two pumps was monitored at the output of the fiber. Then the feedback circuit targeted minimization of the sum of the average powers of the two idlers, which would imply that the length over which the two pump waves were cross-polarized inside the HNLF was maximized. Examples of this nonlinear interaction, when no signals were launched in the HNLF, are displayed in Fig.13 for two different (relative) states of polarization of the two pumps.

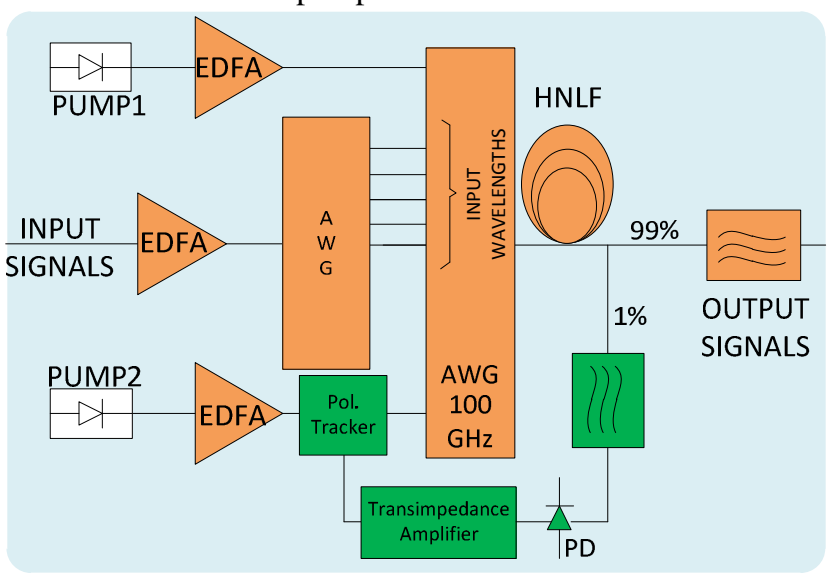

Fig. 14 Modifications performed in the wavelength converter scheme to provide polarization stability. The feedback circuit consists of a dual-frequency filter, a photodiode (PD), a transimpedance amplifier and a polarization tracker.

The required modifications on the WC scheme are shown in Fig. 14. The modified system comprised a polarization tracker, a dual-frequency optical filter, a slow photodiode and a transimpedance amplifier. $1 \%$ of the power at the HNLF output was tapped off and filtered by a dual-frequency square-like filter of 0.1-nm bandwidth for each peak, allowing only the two idlers generated from the pump-to-pump interaction to go through. It is worth mentioning that it was observed that filtering and minimizing the power of only one of the idlers would not lead to the desired alignment between the two pumps. The filtered signal was then detected using the photodetector and the corresponding electrical signal was subsequently amplified by a transimpedance amplifier and used as a feedback signal into the polarization tracker.

Unfortunately, the commercial polarization tracker that we had available at the time of our experiments restricted the maximum optical power allowed at its input to only $20 \mathrm{dBm}$, thereby preventing us to launch the same pump power to the HNLF as before (now only $16.5 \mathrm{dBm}$ per pump was used at the input of the fiber, as opposed to $21.5 \mathrm{dBm}$ in the experiments reported in previous sections). Therefore, the conversion gain obtained at the output of the system was compromised in this instance by $8 \mathrm{~dB}$. (Inverting the order between the EDFA and the polarization tracker in the path of Pump2 in the setup of Fig. 14 would not solve the problem due to the residual PDG of the amplifier used. Indeed, in that configuration the polarization tracker would tend to align the pump polarization to the polarization axis that yielded minimum gain in the EDFA rather than to the polarization that would be orthogonal to the second pump.)

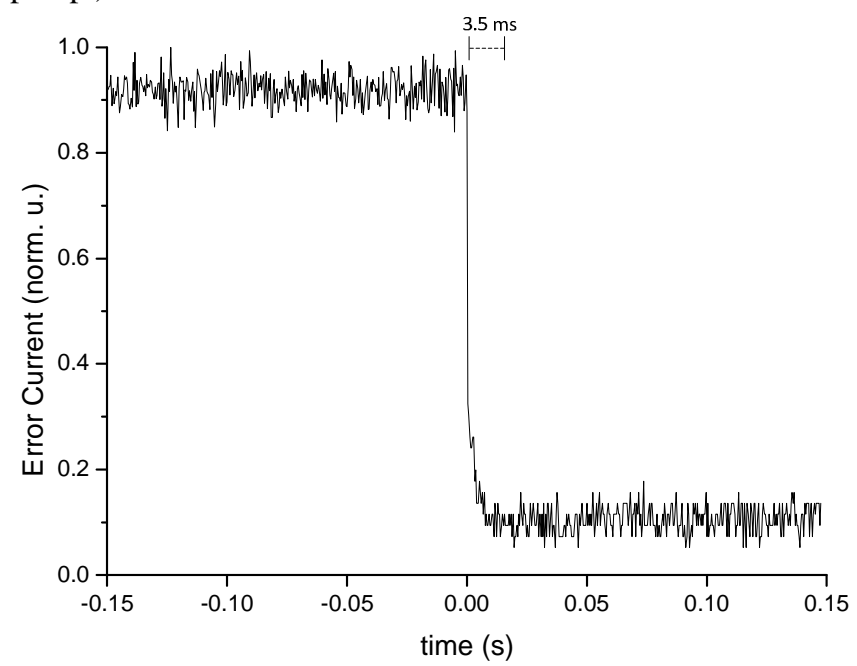

Fig. 15 Experimental characterization of the pump alignment system: response time after switching on the automatic feedback.

The best polarization tracker settings were found to be a fixed step size of $1^{\circ}$ on the Poincaré sphere and an averaging over 15 samples to effectively reduce the noise bandwidth. The bandwidth of the polarization tracker (without averaging) was $500 \mathrm{kHz}$, so that any possible polarization drift could be easily followed with inexpensive discrete elements.

The performance of the feedback circuit was assessed in terms of (a) the response time required to lock to the desired polarization alignment and (b) the stability in maintaining the correct pump polarization alignment when operating the WC. Fig. 15 shows the switch-on time of the feedback circuit. The pumps were initially co-polarized (representing the worst-case scenario) corresponding to a maximum PD current. The figure shows a response time of 3.5 milliseconds, indicating the time 
required by the system to find the desired alignment between the pumps. This time scale is much faster than any polarization drift induced by temperature changes and mechanical vibrations to any of the system components.

Fig. 16 shows the performance of the WC in terms of BER as a function of time (over a period of 150 minutes) when the automatic polarization alignment circuit was either used (red dashed curve) or not (black solid curve). For this characterization a $10 \mathrm{~Gb} / \mathrm{s}$ NRZ OOK signal was used at the input of the WC. Note that when the automatic feedback circuit was not in use, the polarizations of the two pumps in the WC were initially optimized manually using the polarization controllers shown in Fig.1. In contrast, the system was allowed to find the optimum operating point automatically in the case when the feedback system was in place.

As can be seen, in the absence of the automatic alignment system the performance of the converted signal slowly and randomly degraded. Conversely, error-free operation $\left(\mathrm{BER}<10^{-9}\right)$ was continuously maintained throughout the time that the feedback system was operational.

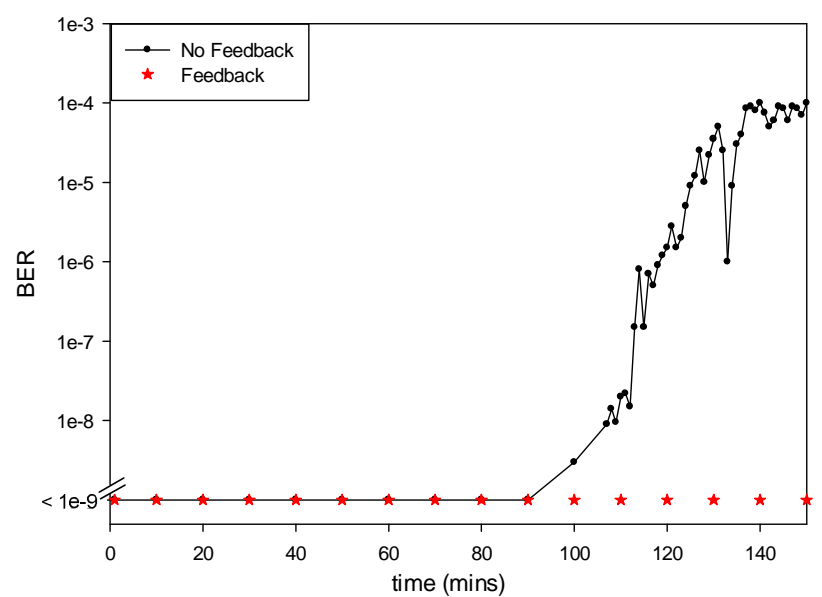

Fig. 16 BER curves as a function of time in absence (black solid line) and presence (red dashed line) of the automatic feedback system.

\section{V.CONCLUSIONS}

A new flexible, modulation-, bit rate- and polarization-independent wavelength conversion scheme based on dual-pump FWM in a HNLF, accommodating $100 \mathrm{GHz}$ grid-aligned signals was presented and characterized. Both in single- and multi-channel operation the WC exhibits a power penalty of $\sim 2.5 \mathrm{~dB}$ as compared to the original signal, a flat conversion gain across its $10-\mathrm{nm}$ bandwidth and a peak-to-peak PDL of $0.64 \mathrm{~dB}$.

The performance of the WC when installed at the mid-point of a transmission experiment was identical to that achieved in laboratory experiments. An optoelectronic system for automatic alignment and stabilization of the relative states of polarization of the two pump signals was proposed and implemented in the WC. While the maximum input power limitation of the polarization tracker used prevented us from achieving the same level of conversion gains as obtained without the automatic feedback circuit, the remaining features of the WC remained unchanged. We believe that our experiments highlight that all-optical wavelength conversion solutions have reached a sufficient level of technological maturity to be considered for deployment in real systems.

\section{ACKNOWLEDGMENTS}

The authors thank the High Performance Networks group of the University of Essex (now at the University of Bristol) for their help during the fiber link commissioning works and OFS Fitel Denmark for providing the HNLF used. The support of JISC and JANET (UK) for the funding and provision of the JANET-Aurora dark-fiber facility is gratefully acknowledged.

\section{REFERENCES}

[1] B. Ramamurthy and B. Mukherjee, "Wavelength Conversion in WDM Networking", IEEE Journal of Selected Areas in Communications, vol. 16, p. 1061-1073, 1998.

[2] J. Strand and G. Li, "Importance of Wavelength Conversion in an Optical Network", Optical Networks, vol. 2, p. 33-39, 2001

[3] K. K. Y. Wong, M. E. Marhic, K. Uesaka and L. G. Kazovsky., "Polarization-Independent Two-Pump Fiber Optical Parametric Amplifier", IEEE Photonic Technology Letters, vol. 14, p. 911-912, 2002

[4] B. P.-P. Kuo, E. Myslivets, A. Wiberg, S. Zlatanovic, C.-S. Bres, S. Moro, F. Gholami, A. Peric, N. Alic and S. Radic, "Transmission of $640-\mathrm{Gb} / \mathrm{s}$ RZ-OOK Channel Over 100-km SSMF by Wavelength-Transparent Conjugation", Journal of Lightwave Technology, vol. 29, p. 516-523, 2011

[5] P. Devgan, T. Renyong, V. S. Grigoryan and P. Kumar., "Highly efficient multichannel wavelength conversion of DPSK signals", Journal of Lightwave Technology, vol. 24, p. 3677-3682, 2006

[6] A. H. Gnauck, E. Myslivets, M. Dinu, B. Kuo, P. Winzer, R. Jopson, N. Alic, A. Konczykowska, F. Jorge, J-Y. Dupuy and S. Radic.,"All-Optical Tunable Wavelength Shifting of a 128-Gbit/s 64-QAM Signal", ECOC Th2.F.2, 2012

[7] X. Li, J. Yu and Z. Dong and N. Chi, "Wavelength conversion of 544-Gbit/s dual-carrier PDM- 16QAM signal based on the co-polarized dual-pump scheme", Optics Express, vol. 20, p.21324-21330, 2012

[8] J. Yu, M-F Huang and N. Cvijetic., "In-band Wavelength Conversion of 16x114-Gbps Polarization Multiplexed RZ-8PSK Signals with Digital Coherent Detection", OFC OThS7, 2009

[9] V. J. F. Rancaño, F. Parmigiani, P. Petropoulos and D. Richardson., "100GHz Grid-Aligned Reconfigurable Polarization Insensitive Black-Box Wavelength Converter" OFC JTh2A.19, 2013

[10] G. Zarris, E. Hugues-Salas, N. Amaya, R. Weerasuriya, F. Parmigiani, D. Hillerkuss, P. Vorreau, M. Spyropolou, S. Ibrahim, P. Petropoulos and D. Simeonidou., "Field Experiments With a Grooming Switch for OTDM Meshed Networking," Journal of Lightwave Technology, vol. 28, pp. 316-327, 2010

[11] V. J. F. Rancaño, F. Parmigiani, P. Petropoulos and D. Richardson., "Field Trial Experiment over $1200 \mathrm{~km}$ on a $100 \mathrm{GHz}$ Grid-Aligned Multi-Channel Black-Box Wavelength Converter" OFC OTh1C.2, 2013

[12] L. Grüner-Nielsen, , D. Jakobsen, S. Herstrøm, B. Pálsdóttir, S. Dasgupta, D. Richardson, "Brillouin Suppressed Highly Nonlinear Fibers," ECOC, We.1.F.1, 2012

[13] C. J. McKinstrie, M. Yu, M. G. Raymer and S. Radic "Quantum noise properties of parametric processes, Optics Express 13(13) pp. 4986-5012, 2005

[14] S. Y. Set, R. Girardi, E. Riccardi, B. Olsson, M. Puleo, M. Ibsen, R. Laming, P. Andrekson, F. Cisternino and H. Geiger, "40Gbit/s field transmission over standard fibre using midspan spectral inversion for dispersion compensation", Electronics Letters, vol. 35, pp. 581-582, 1999

[15] C. R. Menyuk and A. Galtarossa, "Polarization Mode Dispersion", Springer, 2005

[16] M. E. Marhic, "Fiber optical parametric amplifiers and related devices", Cambridge University Press, 1st Ed, 2008 
[17] C. D. Poole and R. E. Wagner, "Phenomenological approach to polarization dispersion in long single-mode fibers", Electronics Letters, vol. 22, p. 1029-1030, 1986

[18] S. Takasaka, M. Takahashi, Y. Mimura, M. Tadakuma, R. Sugizaki and T. Yagi., "Polarization Insensitive Arbitrary Wavelength Conversion in Entire C-band using a PM-HNLF”, ECOC Th.9.C.2, 2010

Víctor J. F. Rancaño was born in Gijon, Spain. He graduated in Communications Engineering at University Carlos III, Madrid, Spain and worked as a development engineer on optical network management at Telefonica R\&D and as an optical system engineer at Huawei Technologies for six years. Currently he is working toward the end of his Ph.D. degree in optical communication systems with the Optoelectronics Research Centre, University of Southampton, UK.

Francesca Parmigiani was born in Milan, Italy. She graduated with honours in Electronic Engineering at Politecnico di Milano, Milano, Italy, in 2002, and received the Ph.D. degree in optical communication systems at the Optoelectronics Research Centre (ORC), University of Southampton, U.K in 2006. She is currently a Senior Research Fellow at the ORC. In April 2010 she was awarded a prestigious Postdoctoral Research Fellowships from the Royal Academy of Engineering, in support of her research on the combination of all-optical signal processing and advanced modulation formats. Her research has produced more than 155 papers in journals and conferences in the field of optical communications. Her research interests include ultra-fast all-optical sampling techniques, pulse shaping using specialized fiber Bragg gratings, all-optical nonlinear processing and switching mainly in optical fibers, as well as advanced modulation formats. Dr Parmigiani is a member of the Optical Society of America (OSA).

Prof. Periklis Petropoulos graduated from the Department of Electrical Engineering and Information Technology, University of Patras, Greece in 1995. He received the MSc degree in Communications Engineering from the University of Manchester Institute of Science and Technology, UK in 1996 and the $\mathrm{PhD}$ degree in Optical Telecommunications from the Optoelectronics Research Centre (ORC), University of Southampton, UK in 2000.

Dr. Petropoulos is a Professor of Optical Communications at the ORC. His research interests lie in the fields of optical communications, all-optical signal processing and nonlinear fibre technology. He has participated in several European Union and national research projects in the field of optical communications. His research has produced more than 350 papers in technical journals and conference proceedings, including several invited and post-deadline papers in major international conferences, and holds 6 patents. Dr. Petropoulos is a Senior Member of the Optical Society of America. He has served as member of the Technical Programme Committees for several international conferences, including the European Conference on Optical Communication (ECOC), the Optical Fiber Communication (OFC) conference and the European Conference on Lasers and Electro-Optics (CLEO/Europe). He is the Chair of the "Photonic Subsystems for Digital System Applications” Subcommittee of OFC'2015.

Prof. David J. Richardson joined the Optoelectronics Research Centre (ORC) at University of Southampton, Southampton, U.K., as a Research Fellow in May 1989. He was awarded a Royal Society University Fellowship in 1991 in recognition of his pioneering work on short pulsed fiber lasers. He is now a Deputy Director of the ORC and is responsible for much of the ORC's fiber related activities. His current research interests include amongst others: optical fiber communications, microstructured optical fibers and highpower fiber lasers. He has published more than 700 conference and journal papers during his time at the ORC and produced more than 15 patents. He was one of the cofounders of SPI Lasers Ltd., an ORC spin-off venture acquired by the Trumpf Group in 2008 .

Prof. Richardson is a Fellow of the Optical Society of America, the Institute of Engineering and Technology, and the Royal Academy of Engineering. 